\title{
Gradhiva
}

GRADHI

Revue d'anthropologie et d'histoire des arts

Sismographie des terreurs

\section{La représentation muséale des génocides}

Guérison ou traumatisme réactualisé ?

Museal representations of genocide. Healing or reactivated trauma?

\section{Reesa Greenberg}

Traducteur : Françoise Charon, Reesa Greenberg et Élise Dubuc

\section{OpenEdition}

\section{Journals}

Édition électronique

URL : http://journals.openedition.org/gradhiva/758

DOI : 10.4000/gradhiva.758

ISSN : 1760-849X

\section{Éditeur}

Musée du quai Branly Jacques Chirac

\section{Édition imprimée}

Date de publication : 1 mai 2007

ISBN : 978-2-915133-55-4

ISSN : 0764-8928

\section{Référence électronique}

Reesa Greenberg, «La représentation muséale des génocides », Gradhiva [En ligne], 5 | 2007, mis en ligne le 15 mai 2010, consulté le 01 mai 2019. URL : http://journals.openedition.org/gradhiva/758 ; DOI : 10.4000/gradhiva.758

Ce document a été généré automatiquement le 1 mai 2019.

(c) musée du quai Branly 


\title{
La représentation muséale des génocides
}

\author{
Guérison ou traumatisme réactualisé ? \\ Museal representations of genocide. Healing or reactivated trauma?
}

\section{Reesa Greenberg}

Traduction : Françoise Charon, Reesa Greenberg et Élise Dubuc

1 La représentation de soi dans les musées ethnographiques et nationaux se pose de façon tout à fait particulière lorsqu'il s'agit de la représentation d'un peuple minoritaire d'un pays dont l'histoire comporte des génocides. Quelles sont les possibilités muséologiques, les réalités politiques et les considérations psychologiques dans un tel contexte? Autrement dit, les musées peuvent-ils servir d'instruments de mieux-être, et si oui, dans quel sens et pour qui ?

2 La plupart de mes réflexions se fondent sur la représentation du traumatisme dans les musées de la religion et des cultures juives (Jewish Museums) ou dans les musées des communautés juives d'Europe et d'Amérique du Nord après la Seconde Guerre mondiale. J'apporterai aussi quelques remarques sur le nouveau National Museum of the American Indian (NMAI) de Washington, qui a ouvert ses portes en 2004 (le musée national des Peuples autochtones des Amériques, dorénavant NMAI). Les différences idéologiques entre le NMAI et les musées des communautés juives de la diaspora peuvent éclairer les possibilités et les limites des musées qui répondent à une histoire de génocides dans les pays où les victimes et leurs descendants continuent de cohabiter avec ceux qui les ont persécutés et leurs héritiers.

Il existe des conditions préalables sans lesquelles il est impossible de fonder un musée ethnographique, soit communautaire, soit national, où le peuple représenté peut déterminer en toute liberté le mode de sa représentation. Certaines de ces conditions préalables sont évidentes, mais il est important de les articuler afin de souligner, tant aujourd'hui que par le passé, les limites de la représentation, pour employer l'expression de Saul Friedlander. La première des conditions préalables est la suivante : un peuple sans ou avec un minimum de pouvoir ne peut contrôler ouvertement, sans user de stratégies 
de subterfuge ou de résistance, la manière dont il est représenté. En voici deux exemples, dont le pire est le musée des Juifs envisagé par Hitler comme le musée d'un peuple disparu après leur extermination planifiée. Même si, pendant la Seconde Guerre mondiale, les conservateurs et les spécialistes juifs ont travaillé dans le ghetto de Prague, site du futur musée, ils ne pouvaient en modifier le concept de base, un concept absolument opposé à la notion de mieux-être des Juifs, mais un concept essentiel, d'après l'idéologie des nazis, comme preuve de succès de leur mieux-être à eux.

Après la guerre, les gouvernements européens n'ont aucun désir de rendre visible l'histoire récente du génocide connu sous le nom de Shoah ou Holocauste, au cours duquel les nazis et leurs collaborateurs ont assassiné six millions de Juifs, surtout pas dans un musée ouvert au grand public. Pour les gouvernements européens, les Juifs survivants représentent une menace à l'image privilégiée de leur nation après la guerre, car ils incarnent leur honte et leur culpabilité pendant le conflit. Pour les pays occupés pendant la guerre, leur participation, pour la plupart active mais variable, au génocide des Juifs est une histoire qui s'oppose complètement aux récits qui en font des victimes des nazis et, pour les pays nazis, leur inhumanité reste condamnable. Pendant des décennies, les autorités de tous les niveaux - municipal, provincial, national - préfèrent nier leur histoire de guerre au lieu de l'exposer. La combinaison de ce manque de volonté de la part des gouvernements et de la fragilité des Juifs européens, dont les minorités sont très affaiblies dans chaque pays après la guerre, peut expliquer cette longue attente d'un renouvellement des musées de la religion et des cultures juives en Europe. Le renouveau débute dans les années 1980 dans les édifices existants, synagogues ou hôtels particuliers convertis en musée. L'ouverture du Jewish Museum de Berlin en 2001 dans un nouvel édifice construit par l'architecte Daniel Libeskind, après une décennie de débats intenses sur les rapports du musée avec l'histoire de la ville et avec ses musées, représente une étape importante sur la voie de la guérison.

On peut soutenir qu'il fallait que les partenaires, les Juifs et les États européens, en tirent un sentiment de mieux-être pour que soit possible le renouveau du musée public des communautés juives. Il a fallu un demi-siècle et l'acceptation de la réalité historique pour que l'idée d'assumer la culpabilité du génocide soit considérée comme favorable au mieux-être de la nation. Le processus d'acceptation d'une histoire difficile est proche de ce qui a trait au postcolonialisme pour d'autres musées.

6 Les concepts de nationalisme et de transfert de pouvoir sont inséparables de l'histoire des musées. Transfert qui s'est tout d'abord réalisé de l'aristocratie au peuple, notamment après les révolutions française et russe, quand les musées royaux et privés du Louvre et de l'Ermitage sont transformés en institutions nationales et populaires. Plus récemment, le transfert de pouvoir est survenu lors de la libération des pays colonisés ou des pays dont l'histoire comprend des peuples colonisés. La création et le renouvellement des musées nationaux accompagnent ce transfert de pouvoir. Par exemple, la fondation d'un musée national à New Delhi, capitale nouvelle du nouveau pays qu'est l'Inde, constitué en 1948, est une marque tangible du remplacement des institutions coloniales britanniques. Un autre exemple est l'inauguration du NMAI de Washington en septembre 2004 dans un nouveau bâtiment en face du Capitole, le siège du gouvernement américain. Ce musée est le témoin visible et valable d'un changement de régime de pouvoir, actuel ou désiré, dans les affaires autochtones aux États-Unis. Dans chaque cas, la construction d'un nouveau musée représente un pas sur la voie de la guérison et du mieux-être d'un peuple auparavant opprimé. 
$7 \quad$ Une seconde condition préalable à la représentation d'un peuple minoritaire est son désir de se représenter sur le mode muséal, d'être connu dans ou par un contexte muséal et de participer à la culture publique des musées. Les musées de la religion et des cultures juives établis en Europe avant la Seconde Guerre mondiale, dont le plus vieux a été fondé à Vienne en 1891, représentaient une période riche de possibilités, une période où les Juifs se sentaient acceptés par la société comme citoyens, une période où la discrimination des siècles passés et ses restrictions quant aux lieux qu'ils pouvaient habiter, les professions qu'ils pouvaient pratiquer, les écoles qu'ils pouvaient fréquenter, etc., semblaient disparaitre ou, du moins, diminuer. Pour les Juifs, avoir leur musée représentait leur entrée dans la nation, un statut d'égalité, une marque d'acceptation, un signe de mieux-être dans la société non juive. Pour les Juifs et les pays où ils habitaient, leurs premiers musées sont associés aux concepts de progrès et d'évolution de Darwin, concepts trahis par le destin des Juifs sous le régime nazi, où ils étaient classés comme inhumains, donc sans place dans la hiérarchie nouvelle. La fermeture de tous les musées des communautés juives d'Europe par les nazis a causé une rupture dans leur histoire, une rupture que le génocide, son ampleur et les raisons déjà décrites ont fait durer presque un demi-siècle.

Dans son livre Trauma and Recovery: The aftermath of violence-from domestic abuse to political terror, Judith Herman aborde les stades de guérison nécessaires pour surmonter la perte de pouvoir et la rupture des relations causées par les traumatismes. Elle parle du besoin de se souvenir et de faire le deuil, puis du besoin de se relier aux autres. Souvent, le rétablissement des liens se produit avec la découverte de ce que Herman appelle la mission du survivant (1992 : 207-211). Durant l'après-guerre, les musées de la religion et des cultures juives jouent plusieurs rôles, pas toujours dans le même musée et pas toujours pour une même époque. Après l'Holocauste, la complexité des rôles de ces musées comme centres communautaires et commémoratifs, mines de renseignements et bâtisseurs d'histoire, tout en étant des lieux d'éducation conçus pour combattre la discrimination, offre plusieurs possibilités de guérison et de mieux-être.

Comme le dit Felicitas Heimann-Jelinek, conservatrice au nouveau Jewish Museum de Vienne: "Après Auschwitz, le retour à l'ancienne tradition classique des musées est impossible. » (1996: 130) Quelles sont alors les nouvelles formes que prennent les musées des communautés juives après l'Holocauste? Chacun d'eux est aux prises avec la manière de représenter la dévastation de l'histoire récente et de la déplorer. La plupart présentent une collection d'artefacts judaïques et une chronique de l'histoire juive comme moyens d'aborder le traumatisme de l'Holocauste.

10 Les musées traditionnels conservent de larges collections d'objets, mais, dans les musées de la religion et des cultures juives d'après-guerre, les artefacts sont présentés comme des allusions indirectes à l'Holocauste, comme s'ils étaient sauvés ou restitués des mains nazies. Les artefacts sont habituellement exposés conformément aux cycles des rituels juifs et comme un trésor pour mettre en valeur la richesse de la culture juive et ce qui a été perdu. Moins souvent, comme dans l'atrium du Jewish Museum de Vienne, la perte est évidente, comme le souligne la scénographie dépouillée et frontale de l'exposition de la collection Max Berger. Ici, les étagères en verre confèrent à l'ensemble un caractère de dématérialisation et les objets flottent comme des fantômes. Qu'elles soient abondantes ou minimales, les expositions d'artefacts judaïques ont une double fonction: elles sont le moyen de commémorer les six millions de Juifs assassinés sans inhumation et le rappel tangible aux Juifs survivants que tout n'a pas été perdu. Comme telles, ces expositions 
officielles sont des manifestations communautaires d'autoguérison et des exemples de l'importance du zakhor, le souvenir, dans la foi juive.

11 Les expositions de collections judaïques servent aussi à faire connaitre la religion juive aux non-Juifs. La plupart des visiteurs des musées des communautés juives ne le sont pas. Ce sont souvent des écoliers. Ils arrivent avec peu ou pas de connaissances de ces communautés, de leurs rituels ou de leur histoire. Les expositions d'objets religieux et laïcs sont des outils pédagogiques qui servent à corriger les stéréotypes négatifs et les faux renseignements dans une tentative de contrer les préjugés. Selon le modèle de Herman, ces expositions sont des manifestations de la mission du survivant et du désir de créer un lien. Elles sont aussi des moyens de ce que j'appelle « la guérison de l'autre ». Le désir d'atténuer les préjugés par l'éducation est un exemple de la foi juive dans le tikkun olam, la réparation du monde.

12 Par ailleurs, dans ces musées, on se souvient explicitement de l'Holocauste. Parfois, sa mention prend la forme d'œuvres d'art, comme la sculpture Holocauste de George Segal au Jewish Museum de New York et certaines sections de Remembrance/Renewal, l'œuvre murale réalisée en 1995 par Nancy Spero à Vienne. Parfois, comme dans le Jewish Museum de Vienne, tout ou partie du musée devient un monument à ce qui a été perdu dans l'Holocauste (Greenberg 2003b). Le plus souvent, on y raconte les faits marquants de l'histoire juive et de l'Holocauste sur le mode chronologique. Dans les musées de la religion et des cultures juives d'Allemagne et d'Autriche, la présentation de ce récit offre peu ou pas d'espoir. C'est particulièrement vrai dans les musées de Francfort et de Berlin, où les traumatismes de l'histoire juive sont racontés suivant un parcours en boucle (Greenberg 2000).

13 Le Jewish Museum de Francfort débute son parcours circulaire par l'histoire des persécutions des Juifs dans cette ville au Moyen-Âge et le termine par un compte rendu de l'Holocauste. La section traitant de l'Holocauste comprend deux salles. La première en parle avec des mots et des photographies sur des murs entourant une structure en treillis métallique pareille à une prison et montée au centre d'une salle obscure. Ici, faits et sentiments sont conjugués. Dans la deuxième salle, on voit des photographies et des films sur les camps de concentration, des livres avec les noms de tous les Juifs de Francfort déportés et des drapeaux américain et israélien. L'absence du drapeau allemand et une seule mention brève de la présence juive dans cette cité après 1945 laissent entendre qu'il est impossible pour la communauté juive de vivre à Francfort après l'Holocauste. Le dernier élément muséographique que les visiteurs voient en quittant l'étage principal du musée est un mur commémoratif où sont inscrits les noms des Juifs de Francfort assassinés durant l'Holocauste.

14 De même, pour entrer et sortir des principales sections du Jewish Museum de Berlin, il faut emprunter (successivement) les trois corridors souterrains d'exposition, espaces sombres, en pente, aux murs angulaires, conçus par Libeskind et qui se consacrent chacun à un aspect de l'Holocauste. Même si le Jewish Museum de Berlin propose une vaste section sur la vie juive dans l'Allemagne d'après-guerre, les visiteurs doivent repasser par les sections sur l'Holocauste de la principale exposition et, de nouveau, traverser les corridors de l'Holocauste créés par Libeskind pour quitter le musée.

15 Quand on brise la chronologie, le sentiment d'espoir se fait plus grand. De 1987 à l'automne 2004, les expositions du Jewish Historical Museum d'Amsterdam étaient organisées plutôt par thèmes que selon la chronologie. L'Holocauste était représenté peu après le début du parcours et sans theâtralité, le plus simplement possible. Plusieurs des 
autres expositions thématiques intégraient des objets et des photographies contemporaines qui permettaient aux visiteurs de saisir la continuité de la vie juive, malgré la perte de $75 \%$ de la communauté juive hollandaise. Au musée d'art et d'histoire du Judaïsme, à Paris, le récit photographique et la chronologie de l'Holocauste étaient, à l'origine, présentés sur une mezzanine suspendue à la fin du parcours. Aujourd'hui, il s'agit de la première et de la seule exposition que l'on voit avant de monter le vaste escalier qui mène à l'installation permanente. Pour le meilleur ou pour le pire, pendant leur visite de ce magnifique musée, les visiteurs peuvent ignorer l'Holocauste et se concentrer uniquement sur les splendeurs et les réalisations passées des Juifs (Greenberg 2003a).

En mettant l'accent sur le souvenir du génocide connu sous le nom d'Holocauste et en le faisant connaître, les musées de la religion et des cultures juives d'Allemagne et d'Autriche s'apparentent à un nouveau type de musée des communautés juives d'aprèsguerre, le musée de l'Holocauste. Contrairement aux premiers, les musées de l'Holocauste racontent une seule histoire, celle de l'Holocauste. Les musées de l'Holocauste à l'extérieur de l'Europe sont créés par des survivants comme moyen de raconter ce qu'ils ont vécu et ce qui est arrivé à leur communauté, tant à un monde juif qui, de plus en plus, n'a pas vécu l'Holocauste qu'à un monde non juif. Les musées de l'Holocauste créés par les survivants illustrent donc le désir de l'autoguérison et de la guérison de l'autre, et mettent en pratique les préceptes juifs du zakhor et du tikkun olam.

Les musées de l'Holocauste consistent invariablement en une chronologie de l'Holocauste et en un site commémoratif, souvent dans cet ordre. Les premiers musées de l'Holocauste étaient des lieux plutôt petits, mélancoliques et communautaires. Les expositions évoquaient rarement la vie juive d'avant l'Holocauste, la résistance et l'héroïsme juifs, les victimes non juives des nazis ou la survie après l'Holocauste. Même animée par un survivant, la visite de ces musées provoquait, chez plusieurs visiteurs juifs comme moi qui n'ont pas vécu l'Holocauste et chez de nombreux visiteurs non juifs, un éventail de sentiments - pessimisme, colère, frustration - envers ce qui paraissait être une exposition où la condition de victime était la seule accordée aux Juifs européens.

Les musées de l'Holocauste fondés plus récemment, comme le United States Holocaust Memorial Museum («musée américain commémoratif de l'Holocauste»), inauguré à Washington en 1995, et la Holocaust Exhibition, ouverte en 2000 et qui fait partie de l'Imperial War Museum ( " musée impérial de la guerre ») de Londres, sont beaucoup plus grands. Si les conservateurs de ces musées tentent de présenter les expositions les plus complètes possible, où les lacunes des premiers musées de l'Holocauste sont prises en compte, les visiteurs, qui finissent par voir la déchéance et la mort des Juifs, pays après pays, vivent un sentiment d'impuissance par trop réaliste. Malgré la présence des témoignages vidéo de survivants et une section consacrée aux personnes qui ont aidé les Juifs, tant d'horreurs risquent de provoquer un effet d'accablement. Les photographies sont agrandies à l'échelle des murs ; la reproduction des sons de l'époque accentue aussi le réalisme ; les modèles réduits, les films et les descriptions des massacres et des camps de concentration laissent peu de place à l'imagination. Quand les visiteurs s'engagent dans le parcours - qui est obligé, car il n'y a qu'une seule entrée et une seule sortie -, ils éprouvent un sentiment d'étouffement, rehaussé par la mise en place d'expositions denses dans des corridors sombres et étroits. Cette forme de reconstitution historique est maintenant adoptée par les plus petits musées de l'Holocauste, comme le centre commémoratif de l'Holocauste de Montréal, récemment rénové. 

ma part qu'il y a plusieurs façons de raconter une histoire. Malgré les tentatives réfléchies de contrôler le rythme et les révélations durant une visite, la quantité de documents d'archives et la manière dont ils sont présentés dans plusieurs musées des communautés juives et de l'Holocauste risquent de réactualiser le traumatisme. Peut-être pas chez les derniers survivants de l'Holocauste, qui estiment fondamental de documenter cette histoire aussi complètement que possible alors que des témoins sont encore en vie. Les survivants sont passionnément convaincus que l'information publique agit comme un antidote contre le racisme et la négation de l'Holocauste. Ils ont raison jusqu'à un certain point. Les survivants canadiens de l'Holocauste ont joué un rôle fondamental dans la production des représentations qui ont conduit à l'adoption de la loi sur la propagande haineuse au Canada en 1967. Mais la multiplication des musées de l'Holocauste ne diminue pas la négation de l'Holocauste ni, malheureusement, les génocides. Pour toutes ces raisons, certains estiment que la visite d'un musée de l'Holocauste n'est ni une expérience de guérison, ni un appel à l'action.

Les capitales et les grandes villes clés, comme Montréal, Dallas, Washington et Londres, qui possèdent des musées de l'Holocauste très élaborés, n'ont pas de grands musées publics sur la religion et les cultures juives pour contrebalancer la représentation de l'histoire juive millénaire par un seul événement génocidaire. Il y a plusieurs dangers, tant pour les communautés juives que non juives, à présenter un portrait aussi limitatif des Juifs et à mettre tant d'énergie et d'argent dans un nombre toujours plus grand de musées de l'Holocauste. En se concentrant ainsi sur un seul événement passé, même cataclysmique, beaucoup trop d'histoires importantes et fascinantes au sujet du judaïsme et des communautés juives locales et contemporaines sont passées sous silence, tels l'élargissement du rôle des femmes dans le judaïsme après les années 1960 ou l'évolution démographique des communautés juives. Ainsi, la communauté juive de Montréal, la plus ancienne au Canada, était anglophone, ashkénaze et d'origine européenne ; aujourd'hui, elle comprend plus de $50 \%$ de francophones, des Juifs sépharades d'Afrique du Nord et du Moyen-Orient, aux coutumes très diverses. Ou l'histoire de l'influence des technologies, comme Internet, en tant qu'agents de changement et de renaissance, ou celle des combats d'une minorité pour survivre. Les trois grandes menaces à la survie du judaïsme aujourd'hui sont l'absence d'une vision unificatrice comprenant des buts communs orientés vers l'avenir, l'assimilation et les mariages mixtes. Aucune de ces questions n'a de visibilité dans les musées de l'Holocauste, qui se concentrent sur ce sujet comme seul véhicule de l'identité juive. La perspective est très différente s'il s'agit de la survivance du judaisme ou de la survivance des Juifs.

Le NMAI a mis en œuvre une vision muséale différente. Au lieu de souligner les nombreux traumatismes de l'histoire des autochtones, les conservateurs ont utilisé trois expositions permanentes thématiques, Our Universes: Traditional Knowledge Shapes the World («Nos univers : la connaissance traditionnelle façonne le monde »); Our Peoples: Giving Voice to Our Histories (« Nos peuples : raconter nos histoires »); et Our Lives: Contemporary Life and Identity ( Nos vies : vie et identité contemporaine »), pour mettre l'accent sur ce que le théoricien nord-amérindien Gerald Vizenor appelle la « survivance ». Bien qu'il écrive en anglais, Vizenor a adopté le mot français parce qu'il croit que «la survivance est un sentiment dynamique de présence ». Pour lui, «les histoires de survivance autochtone sont des histoires de renoncement à la domination, à la tragédie et à la condition de victime » (1999: VII). Comme équivalent français du concept de «survivance » employé 
par Vizenor, je propose celui de résilience du psychiatre français Boris Cyrulnik, comme mécanisme fondamental de guérison après le traumatisme (2003).

$\mathrm{Au}$ NMAI, les tragédies vécues par les autochtones après le contact sont racontées et commémorées, mais l'accent est placé sur l'enseignement des coutumes et des langues indiennes aux jeunes, l'adaptation à la société contemporaine et la lutte pour l'équité. En d'autres termes, le NMAI est orienté vers le futur plutôt que vers le passé. Le musée baigne dans le passé, mais celui-ci est présenté comme une force positive de l'identité autochtone contemporaine. Les images des enfants et de la vie d'aujourd'hui abondent dans le musée, tout comme la technologie contemporaine, et ce pour indiquer que les cultures amérindiennes sont des cultures vivantes. Le rythme et la sonorité des différentes langues dans les vidéos dynamisent les expositions. De jeunes guides de différentes nations animent les visites de groupes composés principalement de non autochtones. Pour souligner leur diversité, les nations amérindiennes ont choisi d'être représentées par plusieurs genres d'expositions, mais celles-ci comprennent toutes des photographies, des films ou des vidéos couleur comme marques d'appartenance à la société contemporaine. Malgré les accusations d'insuffisance militante envers les injustices passées et actuelles portées par l'American Indian Movement (AIM), les conservateurs ont choisi une approche positive. Il se dégage du NMAI un sentiment général de solidarité, de transformation, de persévérance et de possibilité contemporaines, des aspects essentiels du mieux-être de peuples qui négocient un avenir différent.

Pourquoi les musées de la religion et des cultures juives n'ont-ils pas adopté ce modèle ? Le génocide est trop récent, les pertes trop grandes, la reconnaissance trop tardive, les survivants trop prédominants et la peur d'une répétition trop élevée. Pour l'instant, la guérison et le mieux-être dans les musées des communautés juives de la diaspora sont inséparables du traumatisme de l'Holocauste. Peut-être que dans la prochaine étape des musées de la religion et des cultures juives d'après l'Holocauste une vision différente sera proposée, celle du mieux-être, une vision qui saura intégrer plus étroitement traumatisme et résilience.

\section{BIBLIOGRAPHIE}

CYRULNIK, Boris

2003 La Résilience ou comment renaître de sa souffrance. Paris, Fabert.

GREENBERG, Reesa

2000 « Die Ästhetik des Traumas: Zur Darstellung des Holocaust in Jüdischen Historischen Museen », in Gabi Dolff-Bonekämper et Edward van Voolen, éds., Denkmale und kulturelles Gedächtnis nach dem Ende der Ost West-Konfrontation. Berlin, Jovis : 227-238.

2003a « Jews, Museums and National Identities », Ethnologies 24/2 :125-137. 
2003b « The Jewish Museum, Vienna: A Holographic Paradigm for History and the Holocaust », in Shelley Hornstein et Florence Jacobowitz, éds., Image and Remembrance: Representation and the Holocaust. Bloomington, Indiana University Press : 235-250.

HEIMANN-JELINEK, Felicitas

1996 « Memoria, Intelligentia, Providentia »,in Felicitas Heimann-Jelineck et Hannes

Sulzenbacher, éds., Jewish Museum Vienna. Vienne, The Jewish Museum Vienna : 122-130.

HERMAN,Judith

1992 Trauma and Recovery: The aftermath of violence-from domestic abuse to political terror. New York, Basic Books.

VIZENOR, Gerald

1999Manifest Manners: Narratives on Post-Indian Survivance. Lincoln, University of Nebraska Press : vii.

\section{RÉSUMÉS}

Une comparaison de la représentation du traumatisme dans les musées de la religion et des cultures juives après la Seconde Guerre mondiale et au National Museum of the American Indian (NMAI) de Washington, créé en 2004, éclaire les possibilités et les limites des musées qui répondent à une histoire de génocides dans les pays où les victimes et leurs descendants continuent de cohabiter avec ceux qui les ont persécutés et leurs héritiers. Le pouvoir et le désir de se représenter sur le mode muséal sont prolongés par une discussion sur des concepts associés au traumatisme - l'autoguérison, zakhor (se souvenir), tikkun olan (reparer le monde), l'orientation temporelle, la survivance et la résilience - en lien avec leurs manifestations muséologiques.

A comparison of how trauma has been represented in Jewish and Holocaust museums since the Second World War and the National Museum of the American Indian (NMAI) in Washington, 2004, highlighting the possibilities and limits of museums when representing the history of genocides in countries where victims and their descendants continue to live alongside perpetrators and their heirs. The power and the desire to represent oneself on the museal platform are examined, followed by a discussion of concepts associated with trauma [selfhealing, zakhor (remembrance), tikkun olam (repairing the world), temporal considerations, survival, and resilience] in the context of museological representations.

\section{INDEX}

Mots-clés : autochtone, génocide, Holocauste, Juif, musées

Keywords : First Nations, genocide, Holocaust, Jewish, Museums

\section{AUTEURS}

\section{REESA GREENBERG}

Reerden@cs.com. Université de York, Toronto - Université de Concordia, Montréal - Université de Carleton, Ottawa 